\title{
METABOLIC CONTROL FOR MAINTAINING NAD IN RATS FED ON TRYPTOPHAN-LIMITING AMINO ACID DIETS
}

\author{
Katsumi Shibata, ${ }^{1}$ Kazumi MotooKA, ${ }^{1}$ Kiku Murata, ${ }^{1}$ \\ and Kazuo IWAI ${ }^{2}$ \\ ${ }^{1}$ Food and Nutrition Laboratories, Faculty of Domestic Science, \\ Teikoku Women's University, Moriguchi, Osaka 570, Japan \\ ${ }^{2}$ Laboratory of Nutritional Chemistry, Department of Food Science and Technology, \\ Faculty of Agriculture, Kyoto University, Kyoto 606, Japan
}

(Received July 29, 1980)

Summary The effect on the tryptophan-NAD pathway of feeding rats on a diet containing an amino acid mixture simulating rice protein plus the limiting amino acids, $0.4 \%$ of lysine $\cdot \mathrm{HCl}, 0.2 \%$ of threonine, $0.1 \%$ of valine, $0.2 \%$ of isoleucine, $0.1 \%$ of methionine and $0.1 \%$ of histidine $\cdot \mathrm{HCl}$ (basal diet + amino acids), was investigated using male weanling rats of the Sprague Dawley strain, and compared with the reference (basal diet), which did not contain the supplement of the limiting amino acids. These two diets were niacin-free. The weight gain in the "basal diet + amino acids" group was higher than that in the "basal diet" group in the early stage and the food intakes of rats in both groups were almost the same. Consequently, tryptophan intake per $\mathrm{g}$ rat body weight in the former group was lower than that in the latter group. Nevertheless, free tryptophan, total niacin and NAD content per $g$ of liver and kidney of both groups were almost the same and remained constant during this experimental period. This was attributed mainly to the fact that tryptophan oxygenase [EC 1.13.11.11] and quinolinate phosphoribosyltransferase [EC 2.4.2.19] activities of organs in the "basal diet + amino acids" group were higher, and urinary excretion of tryptophan and its metabolites in the group was smaller than that in the "basal diet" group in the early stage. In the middle stage, tryptophan intake per $g$ of rat body weight in both groups fell to half the level of the early stage. Nevertheless, NAD content was maintained at a constant level, urinary excretion of tryptophan and its metabolites was decreased, and picolinate carboxylase [EC 4.1.1.45] activity of liver dropped. These results indicate that a strict regulation mechanism must exist for maintenance of NAD content of liver and kidney of rat for at least the 54-day feeding of these incomplete diets. Keywords tryptophan, niacin, tryptophan-NAD pathway, rat tryp-

\footnotetext{
1 柴田克己, 本岡和美, 村田希久, ${ }^{2}$ 岩井和夫
} 
tophan oxygenase, rat quinolinate phosphoribosyltransferase, rat picolinate carboxylase, NAD content, rice protein

The ratio of the essential amino acids in the daily diet is important along with other nutrients for growth and maintaining the healthy biological condition of animals, including humans. Between one-half and two-thirds of the daily protein intake of Asians comes from rice protein. The amino acid composition of cereals including rice protein is not complete. Therefore, many studies on amino acids supplemental to rice or rice proteins have been reported. It has been proved that the limiting amino acids of rice protein are lysine and threonine. The amount of these amino acids to be added to a rice diet, and the desirability of adding a third and fourth amino acid have not yet been clarified. Yoshida(1) reported that rats fed on a diet including an amino acid mixture simulating rice protein, plus lysine, threonine, isoleucine, valine, methionine, and histidine, without niacin grew faster for the first 7 days than the control group (fed on the diet with an amino acid mixture simulating rice protein), then suddenly stopped growing and began to lose weight rapidly. This condition was soon reversed by addition of tryptophan or niacin. Therefore, Yoshida (1) considered that the addition of the limiting amino acids to the amino acid mixture simulating rice protein was favorable for body protein synthesis, and as a result, the flow from tryptophan to NAD must be decreased. However, biochemical changes of the tryptophan-NAD pathway have not been studied in such conditions. Accordingly, we investigated the effect on the tryptophan-NAD pathway of supplementation of rice protein with the limiting amino acids. Changes in body weights of rats in both groups in our study showed a pattern similar to that reported by Yoshida. However, the weight changes occurred around the 50th day instead of the 7th day in the "basal diet + amino acids" group. Moreover, an interesting result was observed on biochemical regulation of the tryptophan-NAD pathway for maintaining NAD content of liver and kidney during the 54-day feeding. The experiment and the results are described here.

\section{MATERIALS AND METHODS}

Animals. Sixty male weanling rats of the Sprague Dawley strain (3 weeks, body weight $40-50 \mathrm{~g}$ ) were obtained from J.C.L. Each rat was kept in an individual wire-bottomed cage and food and water were supplied ad libitum. The compositions of the diets fed are given in Tables 1 and 2 . All rats were fed on the complete amino acid diet, "diet I" shown in Table 1 for the first 7 days, and distributed into two groups. They were fed on "basal diet" and "basal diet + amino acids" shown in Table 2 for 54 days.

Chemicals. Amino acids were obtained from Kyowa Hakko Co., Ltd. Maize starch and maize oil were purchased respectively from Nichiden Kagaku Co., Ltd. and Nippon Shokuhin Kako Co., Ltd. Vitamins and sucrose were acquired from 
Table 1. Composition of the "diet I."

\begin{tabular}{lc}
\hline \multicolumn{1}{c}{ Ingredient } & $(\mathrm{g} / \mathrm{kg}$ diet $)$ \\
\hline Amino acid mixture ${ }^{\mathrm{a}}$ & 100 \\
Maize starch & 400 \\
Sucrose & 400 \\
Maize oil & 50 \\
Salt mixture & 40 \\
Vitamin mixture & (niacin-free) \\
Choline $\cdot$ HCl & 10 \\
Vitamin A & 1 \\
Vitamin D & 15,000 I.U. \\
Vitamin E & 1,500 I.U. \\
\end{tabular}

${ }^{a}$ Miyazaki pattern except for $\operatorname{Trp}(0.085 \%)$ was used (12). ${ }^{\text {b }}$ These compositions were based on Harper's salt and vitamin mixtures (13).

Table 2. Compositions of "basal diet" and "basal diet + amino acids."

\begin{tabular}{lcc}
\hline \multicolumn{1}{c}{ Ingredient } & Basal diet & $\begin{array}{c}\text { Basal diet }+ \text { Amino acids } \\
\text { (g/kg diet) }\end{array}$ \\
\hline Amino acid mixture & & 91 \\
Glutamic acid & 91 & 0 \\
Lysine $\cdot \mathrm{HCl}$ & 11 & 4 \\
Threonine & 0 & 2 \\
Valine & 0 & 1 \\
Isoleucine & 0 & 2 \\
Methionine & 0 & 1 \\
Histidine $\cdot \mathrm{HCl}$ & 0 & 1 \\
Maize starch & 0 & 561 \\
Sucrose & 561 & 281 \\
Maize oil & 281 & 20 \\
Salt mixture & 20 & 40 \\
Vitamin mixture ${ }^{\mathrm{b}}$ (niacin-free) & 40 & 2.5 \\
Choline $\cdot \mathrm{HCl}$ & 2.5 & 2 \\
Vitamin A & 2 & 3,000 I.U. \\
Vitamin D & 3,000 I.U. & 300 I.U. \\
Vitamin E & 300 I.U. & 0.6 I.U. \\
\hline
\end{tabular}

a The amino acid composition of rice protein used was based on "The Amino Acid Composition of Food in Japan, Resources Bureau, Science and Technology Agency (1966)." b These compositions were based on Harper's salt and vitamin mixtures (13).

Wako Pure Chemical Industries Ltd. The salt mixture was the product of Tanabe Amino Acids Research Foundation. Quinolinic acid- ${ }^{14} \mathrm{C}$ was purchased from Daiichi Pure Chemicals Co., Ltd. 5-Phosphoribosyl-1-pyrophosphate and ATP 
were obtained respectively from Sigma Chemical Company and Boehringer Mannheim GmbH. 3-Hydroxyanthranilic acid was obtained from Nakarai Chemicals Ltd. Other chemicals were of the highest purity available from commercial sources.

Enzyme assay. Fresh liver and kidney were thinly sliced, mixed with 5 volumes of $50 \mathrm{~mm}$ potassium phosphate buffer at $\mathrm{pH} 7.0$ and homogenized using a glass homogenizer. This homogenate was used as source for each enzyme. Tryptophan oxygenase was assayed by the increase in the reading of kynurenine at $360 \mathrm{~nm}$ (2). The reaction mixture (total volume $3.0 \mathrm{ml}$ ) contained $0.3 \mathrm{ml}$ of $500 \mathrm{~mm}$ potassium phosphate buffer at $\mathrm{pH} 7.0,0.2 \mathrm{ml}$ of $50 \mathrm{~mm}$ tryptophan, $2.35 \mathrm{ml}$ of water, and $0.15 \mathrm{ml}$ of enzyme solution. Incubation was started by the addition of enzyme and carried out at $37^{\circ} \mathrm{C}$ for $1 \mathrm{hr}$. The reaction was stopped by the addition of $2 \mathrm{ml}$ of $15 \%$ metaphosphate. The deproteinized solution was neutralized with $\mathrm{KOH}$, made up to $10 \mathrm{ml}$ with water and measured at $360 \mathrm{~nm}$. For reference, the same procedure was performed omitting tryptophan. Quinolinate phosphoribosyltransferase was assayed by counting the radioactivity of the ${ }^{14} \mathrm{CO}_{2}$ released from the 5phosphoribosyl-1-pyrophosphate-dependent decarboxylation of quinolinic acid-2, $3,7,8-{ }^{14} \mathrm{C}(3)$. The reaction mixture (total volume $0.5 \mathrm{ml}$ ) contained $50 \mu \mathrm{l}$ of $500 \mathrm{~mm}$ potassium phosphate buffer at $\mathrm{pH} 7.0,15 \mu \mathrm{l}$ of $10.9 \mathrm{~mm}$ quinolinic acid-2,3,7,8- ${ }^{14} \mathrm{C}, 50 \mu$ l of $10 \mathrm{~mm}$ 5-phosphoribosyl-1-pyrophosphate, $20 \mu \mathrm{l}$ of $50 \mathrm{~mm}$ $\mathrm{MgCl}_{2}, 265 \mu \mathrm{l}$ of water, and $100 \mu \mathrm{l}$ of enzyme solution. Incubation was started by the addition of enzyme and carried out at $37^{\circ} \mathrm{C}$ for $30 \mathrm{~min}$. The reaction was stopped by the addition of $0.8 \mathrm{ml}$ of $4 \% \mathrm{HClO}_{4}$. Picolinate carboxylase was assayed by the method of Ichiyama et al. (4).

Determination of tryptophan and its metabolites. Fresh liver and kidney were thinly sliced, mixed with 5 volumes of $0.6 \mathrm{M} \mathrm{HClO}_{4}$, homogenized in a glass homogenizer and centrifuged at $3,000 \times g$ for $10 \mathrm{~min}$. The precipitated material was again extracted. The combined supernatant solution was neutralized with $\mathrm{KOH}$ and centrifuged. This neutralized solution was used as substrate for each determination. Rat urine was collected in a flask with a small amount of $\mathrm{HCl}$ and toluene, using the metabolic cage. Free tryptophan was measured by the method of Denckla and Dewey (5). Total niacin was determined by the microbioassay method with Lactobacillus arabinosus ATCC $8014(6)$. Quinolinic acid was decarboxylated by treatment in the autoclave in glacial acetic acid and the decarboxylated product was determined by the microbioassay method(6). NAD was determined using yeast alcohol dehydrogenase(7). $N^{1}$-Methylnicotinamide and $N^{1}$-methyl-2pyridone-5-carboxamide were measured by the methods of Nose and Ueda ( 8 ) and Tomabechi (9), respectively. 5-Hydroxyindole-3-acetic acid was measured by the method of Udenfriend et al.(10).

Statistical analysis. Statistical analysis was carried out using analysis of variance and Student's $t$ test. 


\section{RESULTS}

\section{Changes in body weight}

Figure 1 shows the weight gains and the food intakes of rats fed on "basal diet" and "basal diet + amino acids." Rats of both groups gained weight linearly for the first 20 days. The average weight gain of the rats fed on "basal diet" was $20 \mathrm{~g}$ and that of the rats fed "basal diet + amino acids" $40 \mathrm{~g}$. After the 20 th day, body weights of rats of both groups varied irregularly. After the 50th day, the weight of the rats in the "basal diet + amino acids" group dropped abruptly, but not that of the rats in the "basal diet" group. No difference in food intake between the two groups was observed, which was decreased by about half after the 30th day. Accordingly, tryptophan intake of both groups was decreased to the same extent, compared to the early stage. Since these diets are niacin-free, all NAD is synthesized from tryptophan. Therefore, the amount of tryptophan intake per $g$ of body weight was calculated. These values are shown in Fig. 2. The amount of tryptophan intake in the "basal diet + amino acids" group was lower than that of the "basal diet" group until the 30th day.

\section{Tryptophan and its metabolites in liver and kidney}

The amount of free tryptophan in liver and kidney of both groups is presented in Fig. 3. Tryptophan intake decreased after the middle stage, while the amount of free tryptophan in liver and kidney of both groups was only slightly decreased and no difference between the two groups was observed during the experiment.

The amount of total niacin in liver of both groups was constant at about $0.5 \mu \mathrm{mol}$ per $\mathrm{g}$, as shown in Fig. 4A. In kidney also, there was no difference between the two groups and the level throughout the experiment was constant at

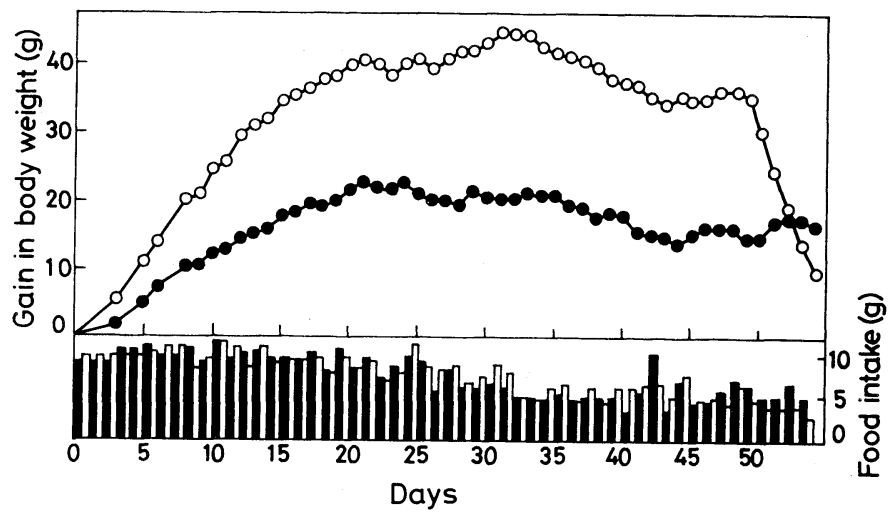

Fig. 1. Weight gain in rats fed on "basal diet" and "basal diet + amino acids." The compositions of "basal diet" and "basal diet + amino acids" are shown in Table 2. Closed column and open column indicate food intakes of "basal diet" group and "basal diet + amino acids" group, respectively, and closed circle and open circle depict weight gains of "basal diet" group and "basal diet + amino acids" group.

Vol. 26, No. 6, 1980 


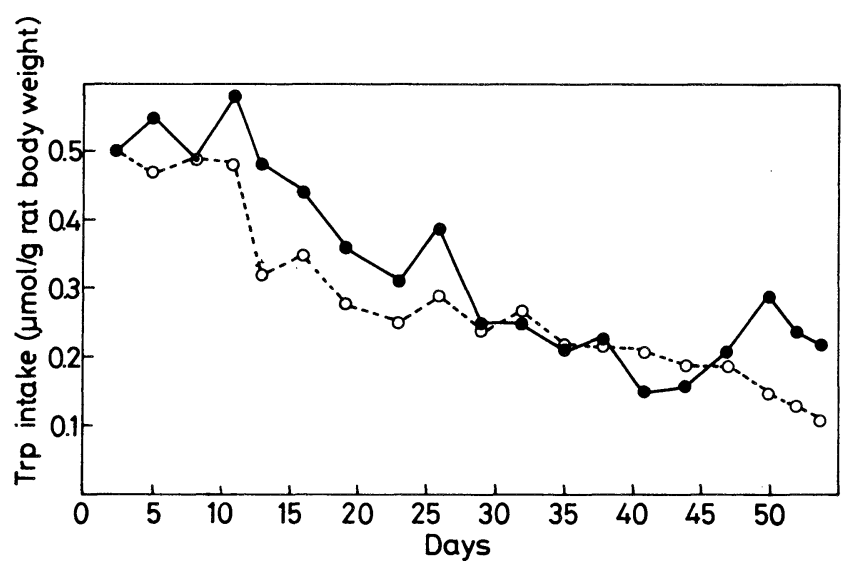

Fig. 2. Changes in tryptophan intakes of rats fed on "basal diet" and "basal diet + amino acids." Amount of tryptophan intake per $\mathrm{g}$ of rat body weight was calculated from food intake and rat body weight. Closed circle and open circle indicate tryptophan intakes per g of rat body weight of "basal diet" group and "basal diet + amino acids" group, respectively.

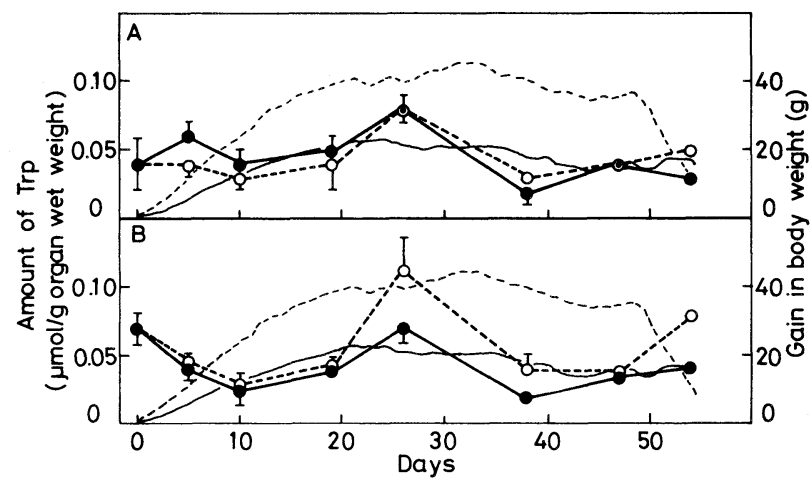

Fig. 3. Changes in free tryptophan content of liver (A) and kidney (B) of rats fed on "basal diet" and "basal diet + amino acids." Rats were anaesthetized with ether. The abdomen was opened by a midline incision and liver and kidney were taken out immediately, washed with cold $0.9 \% \mathrm{NaCl}$ and mixed with 5 volumes of $0.6 \mathrm{M}$ $\mathrm{HClO}_{4}$. Other treatments are described in MATERIALS AND METHODS. Each value except for that of the 47 th ( 3 rats) and 54th days ( 2 rats), is the average of 5 rats. Vertical lines indicate standard deviation and closed circle and open circle show the amounts of free tryptophan in liver (A) and kidney (B) of rats fed on "basal diet" and "basal diet + amino acids." Dotted fine line and solid fine line indicate weight gain in rats fed on "basal diet" and "basal diet + amino acids," respectively. The value of day 0 is the value on the 7th day of feeding rats on a complete amino acid diet, "diet I." The difference between the two groups was statistically significant on the following days: $p<0.01$ on the 5th day in liver: $p<0.01$ on the 26th day and $p<0.02$ on the 38 th day in kidney. 


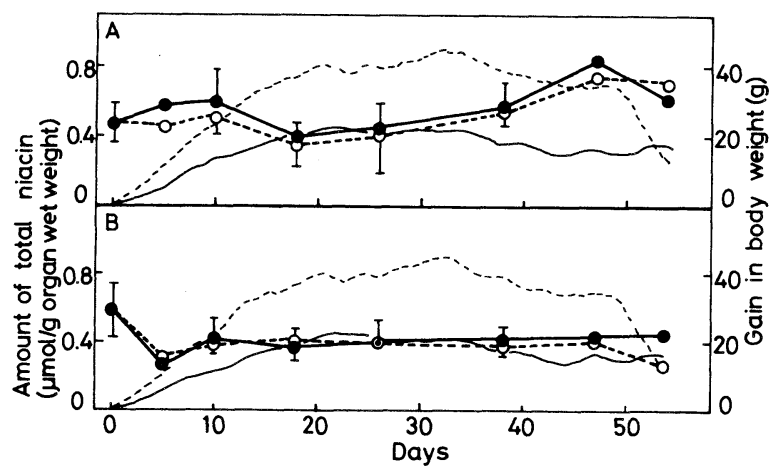

Fig. 4. Changes in total niacin content of liver (A) and kidney (B) of rats fed on "basal diet" and "basal diet + amino acids." For explanation of this figure see the legend in Fig. 3. The difference between the two groups was statistically significant on the following day: $p<0.05$ on the 5 th day in liver.

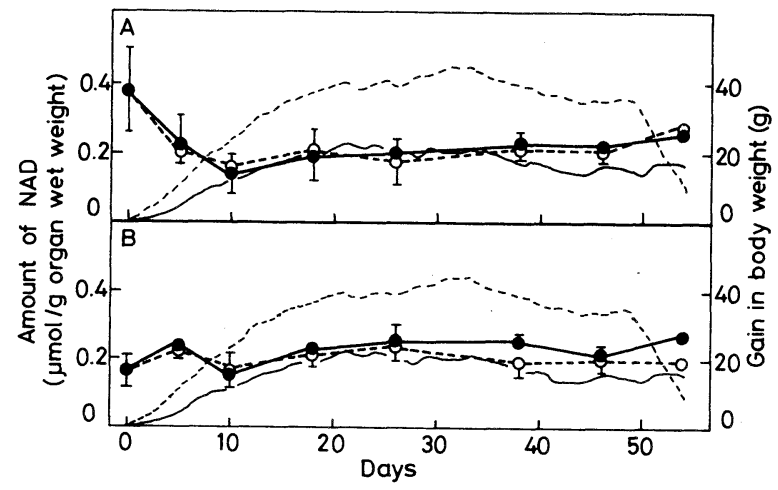

Fig. 5. Changes in NAD content of liver (A) and kidney (B) of rats fed on "basal diet" and "basal diet + amino acids." The explanation of this figure is the same as that of Fig. 3.

$0.4 \mu \mathrm{mol}$ per $\mathrm{g}$, as shown in Fig. 4B.

Quinolinic acid, $N^{1}$-methylnicotinamide and $N^{1}$-methyl-2-pyridone-5carboxamide were detected only in small amounts in liver and kidney of both groups during the experiment.

NAD content is shown in Fig. 5. The NAD content of liver of both groups was decreased from $0.4 \mu \mathrm{mol}$ to $0.2 \mu \mathrm{mol}$ per $\mathrm{g}$ liver wet weight at the 10 th day, after which the amount of NAD in liver remained constant at $0.2 \mu \mathrm{mol}$ per $\mathrm{g}$. The amount in kidney of both groups was almost constant during the experiment $(0.2 \mu \mathrm{mol}$ per $\mathrm{g}$ wet weight). 
Enzyme activities in the tryptophan-NAD pathway

The activity of tryptophan oxygenase, the first enzyme in the tryptophanNAD pathway, is shown in Fig. 6. The activity of the "basal diet + amino acids" group was somewhat higher than that of the "basal diet" group during the experiment except for the 47th day.

The activity of quinolinate phosphoribosyltransferase, which produces the first niacin-active product (nicotinic acid mononucleotide) in liver and kidney, is presented in Fig. 7. In liver, this activity for the "basal diet + amino acids" group was higher than that for the "basal diet" group. However, this activity in kidney was almost the same in both groups.

The activity of picolinate carboxylase in liver and kidney is shown in Fig. 8. No difference between the two groups was observed in liver or kidney. A significant change was the decrease of this activity from the 20th to 40th days in liver of both groups.

\section{Urinary excretions of tryptophan and its metabolites}

The amount of urinary tryptophan, 5-hydroxyindole-3-acetic acid, quinolinic acid, total niacin and $N^{1}$-methylnicotinamide are shown in Figs. 9, 10, 11, 12, and 13, respectively. The amount of each urinary metabolite was increased significantly by the change from "diet I," a complete amino acid mixture, to "basal diet." The increase in urinary levels of tryptophan and its metabolites must be attributed to decreased efficiency of synthesis of body protein from essential amino acids, including tryptophan, because of the poor simulation of rice protein by the amino

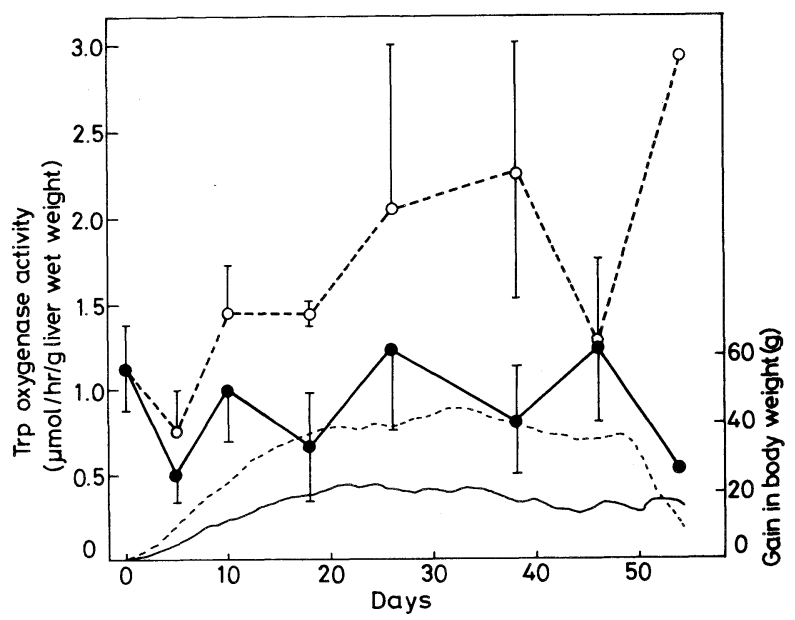

Fig. 6. Changes in the activity of tryptophan oxygenase of rats fed on "basal diet" and "basal diet + amino acids." Experimental conditions are described in MATERIALS AND METHODS. Denotation of this figure is the same as the legend for Fig. 3. The difference between the two groups was statistically significant on the following days: $p<0.05$ on the 10 th, $p<0.001$ on the 18 th and $p<0.01$ on the 38 th days. 


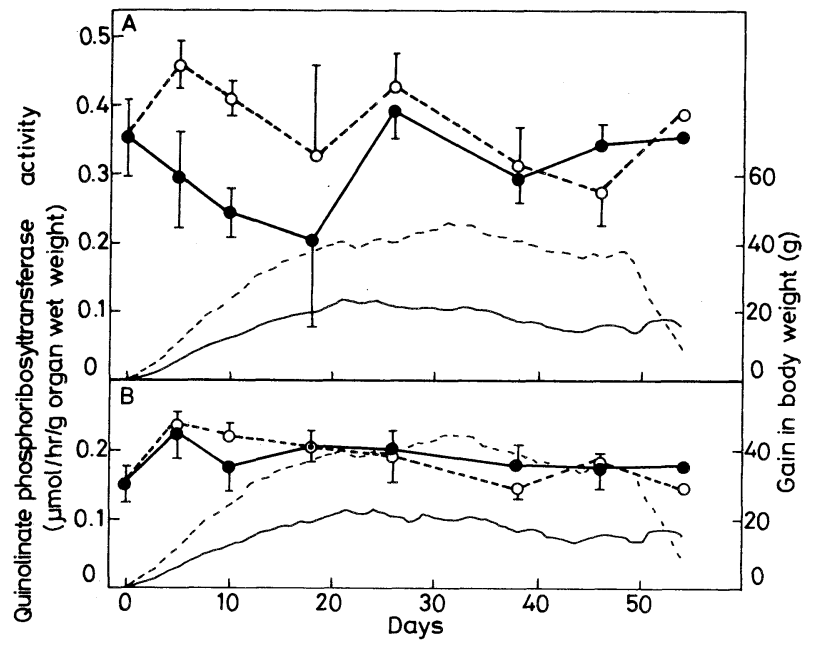

Fig. 7. Changes in the activity of quinolinate phosphoribosyltransferase in liver (A) and kidney (B) of rats fed on "basal diet" and "basal diet + amino acids." Experimental conditions are described in MATERIALs AND Methods. Denotation of this figure is the same as the legend for Fig. 3. The difference between two groups was statistically significant on the following days: $p<0.01$ on the 5 th and $p<0.01$ on the 10th days in liver: $p<0.05$ on the 10th and $p<0.02$ on the 38th days in kidney.

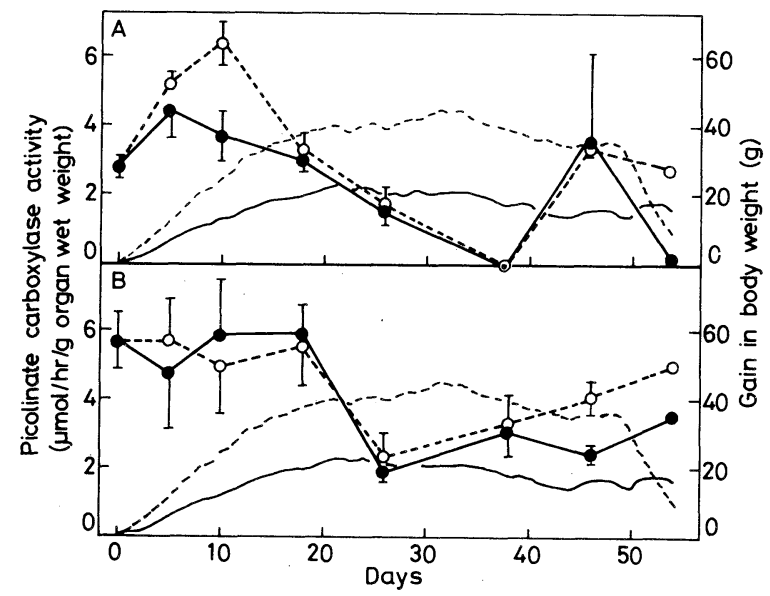

Fig. 8. Changes in the activity of picolinate carboxylase in liver (A) and kindey (B) of rats fed on "basal diet" and "basal diet + amino acids." Experimental conditions are described in MATERIALS AND METHODS. Denotation of this figure is the same as the legend for Fig. 3. The difference between the two groups was statistically significant on the following days: $p<0.001$ on the 10th day in liver: $p<0.01$ on the 47th day in kidney. 


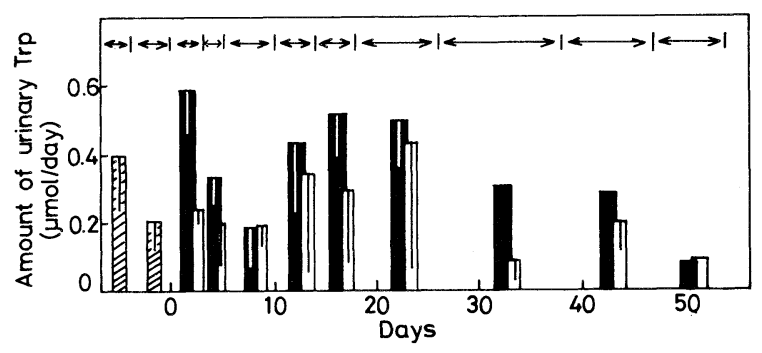

Fig. 9. Changes in urinary tryptophan levels of rats fed on "basal diet" and "basal diet + amino acids." Urines of 3 or 4 rats for each group were collected 11 times as indicated by the arrow. Hatched, closed and open columns show urinary tryptophan of rats fed on "diet I," "basal diet" and "basal diet + amino acids." Other conditions are described in MATERIALS AND METHODS. The difference between the two groups was statistically significant only on days $0-3: p<0.01$.

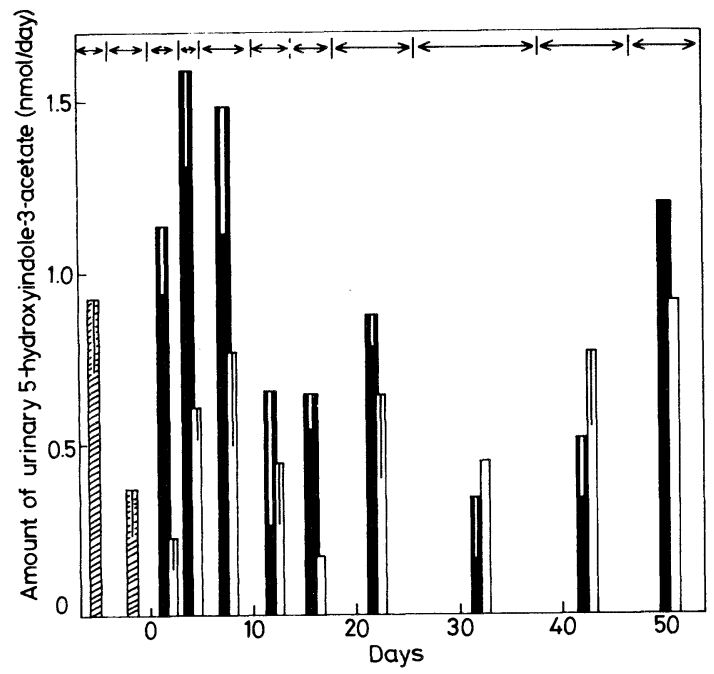

Fig. 10. Changes in urinary 5-hydroxyindole-3-acetic acid levels of rats fed on "basal diet" and "basal diet + amino acid." Experimental conditions are described in Materials And Methods and the legend for Fig. 9. Denotation of this figure is the same as the legend for Fig. 9. The difference between the two groups was statistically significant on the following days: $p<0.001$ for days $0-3, p<0.05$ for days $3-5$ and $p<0.05$ for days $5-10$.

acid mixture of the "basal diet." On the other hand, by the change from "diet I" to "basal diet + amino acids," the level of individual urinary metabolites was not increased as much. The level of each urinary metabolite in the "basal diet" group was higher than that in "basal diet + amino acids" group. The difference was remarkable in the early stage. $N^{1}$-Methylnicotinamide in the "basal diet + amino acids" group was detected only during the first 15 days. $N^{1}$-Methyl-2-pyridone-5carboxamide was not detected in the urine of either group. 


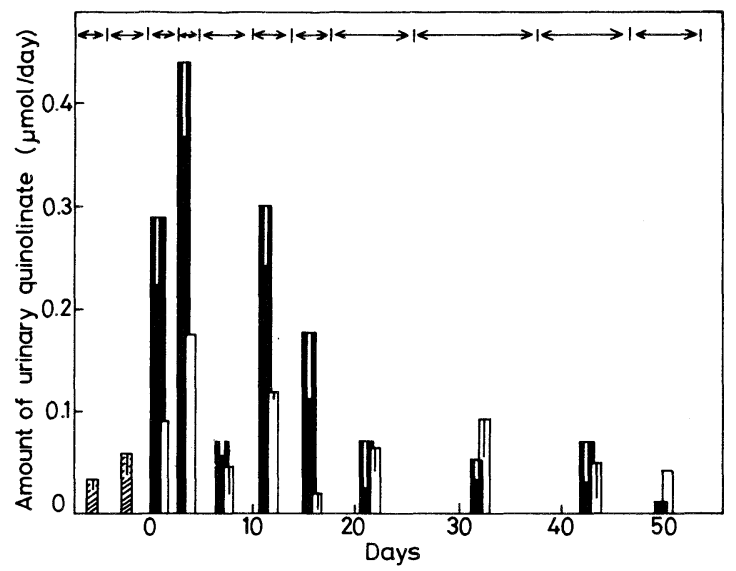

Fig. 11. Changes in urinary quinolinic acid levels of rats fed on "basal diet" and "basal diet + amino acids." Experimental conditions are described in MATERIALS AND Methods and the legend for Fig. 9. Denotation of this figure is the same as the legend for Fig. 9. The difference between the two groups was statistically significant on the following days: $p<0.01$ for days $0-3, p<0.001$ for days $3-5, p<0.02$ for days $10-14$ and $p<0.02$ for days $14-18$.

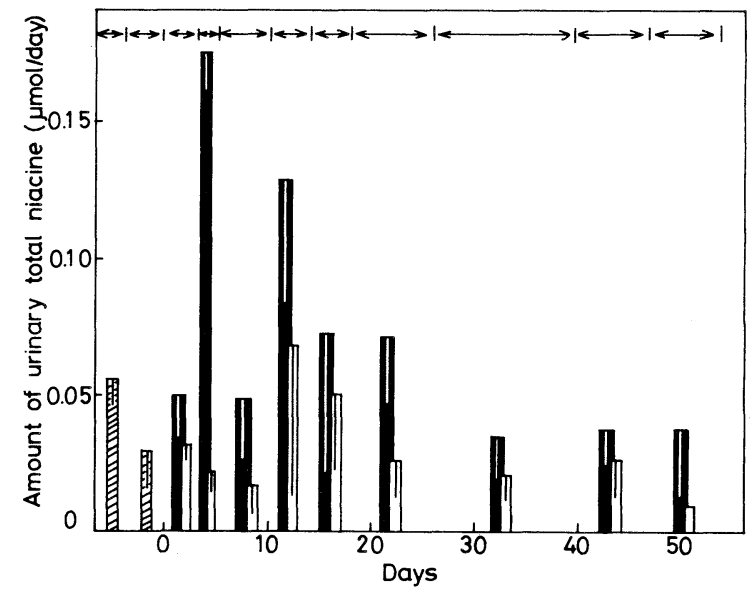

Fig. 12. Changes in urinary total niacin levels of rats fed on "basal diet" and "basal diet +amino acids." Experimental conditions are described in MATERIALS AND Methods and the legend for Fig. 9. Denotation of this figure is the same as the legend for Fig. 9. The difference between the two groups was statistically significant on the following days: $p<0.001$ for days $3-5$ and $p<0.02$ for days 18-26. 


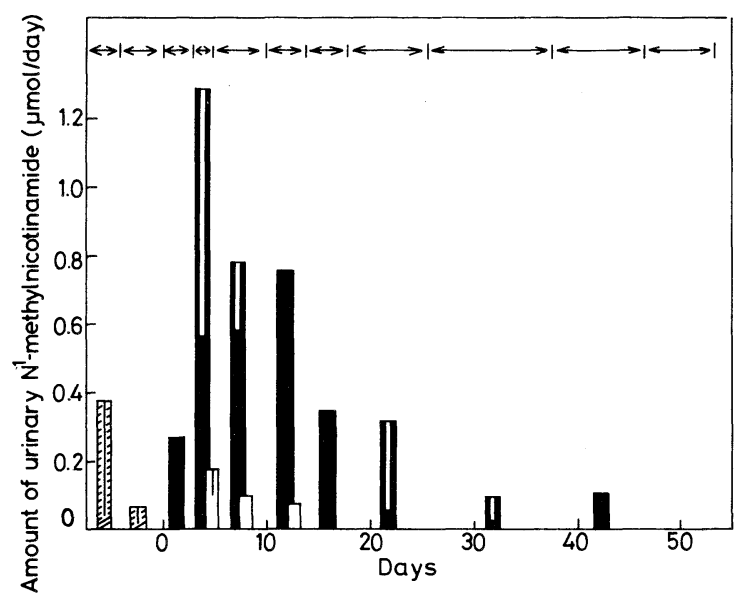

Fig. 13. Changes in urinary $N^{1}$-methylnicotinamide levels of rats fed on "basal diet" and "basal diet + amino acids." Experimental conditions are described in MATERIALS AND METHODS and the legend for Fig. 9. Denotation of this figure is the same as the legend for Fig. 9. The difference between the two groups was statistically significant: $p<0.01$ for days $5-10$.

\section{DISCUSSION}

If the growth of an animal is promoted by supplementation of the rice protein with the limiting amino acids, the other essential amino acids must be more efficiently utilized for body protein. In a feeding test on rats by Yoshida (1), using a diet containing an amino acid mixture (without niacin) simulating rice protein supplemented with the limiting amino acids, the animals showed a sudden decrease of body weight following promotion of growth for 7 days. He considered that the flow from tryptophan to NAD must be decreased by the promotion of growth during the 7 days. In our experiment, a similar sudden decrease of weight of rats were observed after the 50th day of feeding. In our investigation of tryptophan utilization, we measured tryptophan intake per $\mathrm{g}$ of body weight, changes in the activity of key enzymes in the tryptophan-NAD pathway and the amounts of tryptophan metabolites excreted during the 54-day feeding period. The results showed the existence of a strict regulatory mechanism for maintaining NAD at a constant, probably the minimum level. The existence of the biochemical regulation is supported by the following evidence: 1) the activities of tryptophan oxygenase and quinolinate phosphoribosyltransferase in the "basal diet + amino acids" group were significantly higher in the early stage: 2) the activity of picolinate carboxylase in livers of both groups dropped from the middle stage to the last stage: 3) urinary excretion of tryptophan and its metabolites in both groups decreased from the middle to the last stage: 4) $N^{1}$-methylnicotinamide and $N^{1}$ methyl-2-pyridone-5-carboxamide were not detectable in liver and kidney of either 
group. In other words, the activities of the enzymes, tryptophan oxygenase, quinolinate phosphoribosyltransferase and picolinate carboxylase and the system for the urinary excretion of tryptophan and its metabolites must be considered responsible for maintaining NAD at a constant level.

Ghaloupka et al.(11) reported that supplementary tryptophan was first used for protein synthesis in young rats depleted of niacin and subsequently as a precursor of blood pyridine nucleotides. Yoshida(1) also considered that tryptophan was predominantly used for protein synthesis even when niacin was the limiting nutrient. However, the present results indicate that tryptophan favored synthesis of NAD maintained at a constant level, probably the lowest level possible for survival, even when tryptophan was deficient for synthesis of body protein. The reason for the difference in body weight changes of rats in our experiment and that of Yoshida (1) might owe to difference of strain and age of rats used in the two experiments.

The authors express their sincere appreciation to Dr. C. S. Rose for the reading of our manuscript. We are grateful to Kyowa Hakko Co., Ltd., for supplying amino acids, and also to our students for their technical assistance.

\section{REFERENCES}

1) Yoshida, A. (1971): Effect of amino acid supplementation to a rice diet on niacin requirement of rats. Agric. Biol. Chem., 35, 1943-1949.

2) Knox, W. E. (1955): Tryptophan oxidation. A. L-Tryptophan peroxidase from liver, in Methods in Enzymology, Vol. 2, ed. by Colowick, S. P., and Kaplan, N. O., Academic Press, New York, pp. 242-246.

3) Iwai, K., and Taguchi, H. (1973): Distribution of quinolinate phosphoribosyltransferase in animals, plants and microorganisms. J. Nutr. Sci. Vitaminol., 19, 491-499.

4) Ichiyama, A., Nakamura, S., Kawai, H., Honjo, T., Nishizuka, Y., Hayaishi, O., and Senoh, S. (1965): Studies on the metabolism of the benzene ring of tryptophan in mammalian tissues. II. Enzymatic formation of $\alpha$-aminomuconic acid from 3hydroxyanthranilic acid. J. Biol. Chem., 240, 740-749.

5) Denckla, W. D., and Dewey, H. K. (1967): The determination of tryptophan in plasma, liver and urine. J. Lab. Clin. Med., 69, 160-169.

6) Iwai, K., Okinaka, O., and Yokomizo, H. (1967): A uniform medium for microbiological determination of the B-vitamins with various lactic acid bacteria. Vitamins (in Japanese), 35, 387-394.

7) Klingenberg, M. (1974): Nicotinamide-adenine dinucleotides (NAD, NADP, NADH, NADPH) spectrophotometric and fluorometric methods. in Methods of Enzymatic Analysis, Vol. 4, ed. by Bergmeyer, H. U., Academic Press, New York, pp. 2045-2059.

8) Nose, Y., and Ueda, K. (1949): $N^{1}$-Methylnicotinamide no teiryoho (2). Acetone shukugo keikoho. (The determination of $N^{1}$-methylnicotinamide (2). A fluorometric method by acetone condensation.) Vitamins (in Japanese), 2, 247-251.

9) Tomabechi, K. (1952): Studies on the nicotinic acid metabolism (I). A fluorometric determination of 6-pyridone. Vitamins (in Japanese), 5, 463-465.

10) Udenfriend, S., Titus, E., and Weissbach, H. (1955): The determination of 5hydroxyindole-3-acetic acid in normal urine and a method for its assay. J. Biol. Chem.,

Vol. 26, No. 6, 1980 
216, 499-505.

11) Ghaloupka, K. M., Williams, J. N., Jr., Raynolds, M. S., and Elvehjem, C. A. (1957): Relative roles of niacin and tryptophan in maintaining blood pyridine nucleotides, nitrogen balance and growth in adult rats. J. Nutr., 63, 361-375.

12) Miyazaki, M., and Hayakawa, Y. (1965): Shironezumi no seicho ni hitsuyo na aminosan pattern (zokuho) (Reports of The Research Committee of Essential Amino Acids (in Japanese), No. 25, 89.

13) Harper, A. E. (1959): Amino acid balance and imbalance. I. Dietary level of protein and amino acid imbalance. J. Nutr., 68, 405-418. 